\title{
On the six-dimensional Kerr theorem and twistor equation
}

\author{
Bruno Carneiro da Cunha ${ }^{\mathrm{a}}$ \\ Departamento de Física, Universidade Federal de Pernambuco, Recife, Pernambuco 50670-901, Brazil
}

Received: 17 March 2014 / Accepted: 7 April 2014 / Published online: 29 April 2014

(C) The Author(s) 2014. This article is published with open access at Springerlink.com

\begin{abstract}
The Kerr theorem is revisited as part of the twistor program in six dimensions. The relationship between pure spinors and integrable 3-planes is investigated. The real condition for Lorentzian spacetimes is seen to induce a projective property in the space of solutions, reminiscent of the quaternionic structure of the six-dimensional Lorentz group. The twistor equation (or Killing spinor equations generically) also has an interpretation as integrable null planes, and a family of Einstein spacetimes with this property are presented in the Kerr-Schild fashion.
\end{abstract}

\section{Introduction}

The Kerr theorem shows how to generate maximally null integrable submanifolds in flat spacetime. The importance of such a characterization was technical at first, with solutions of Einstein's Equations in the Newman-Penrose formalism in mind [1,2]. Many solutions have been found this way, including the Kerr-Newman solution. For complexified spaces, Hughston and Mason [3] showed how to parametrize these null manifolds in terms of pure spinors and posteriorly related the structure to the integrability of the wave equation $[4,5]$. The same connection was noted by the mathematicians at around the time [6] to provide a higher-dimensional analog of "Bateman's Formula", or Penrose transform. These have been recast in the twistor language by Berkovits and Cherkis [7].

From the physical point of view, the existence of null integrable submanifolds is at the heart of the simplification of the metric structure that happens near the horizon of a black hole, even without the presence of supersymmetry [8,9]. This is a horizon analog of the "peeling theorem" [10], which originally stated that the corrections to the metric in asymptotic flat spacetimes are ranked in order of algebraic specialty, as defined by Petrov. The Petrov classification of the Weyl

\footnotetext{
a e-mail: bcunha@df.ufpe.br
}

tensor sees the latter as a linear application in the space of bivectors. Because of the symmetry of the Weyl tensor, eigenvalues are the outer product of null vectors, dubbed principal null directions. With respect to the peeling theorem, in particular, one can state that the contributions to the metric from charges such as ADM mass and angular momentum allow for the definition of a principal null direction, picking a particular one from the infinite directions of flat spacetime. This fact was used by Penrose to define local conserved charges in Twistor Theory $[11,12]$.

The relationship between null integrable manifolds and spinors have been outlined since its inception [13,14]. In the applications of spinors in General Relativity this connection has always been explicit; however, in applications to supergravity and superstrings the algebraic approach has always been preferred. In four dimensions, the work of van Nieuwenhuizen and Warner [15] built the bridge between the two formalisms by working the conditions for the existence of Killing spinors in the Newman-Penrose formalism.

This letter will try a similar program in six dimensions. We will revisit the Kerr theorem in six dimensions and define helicity spinors by exploring the symmetry of the solutions. Previous work along those lines was conducted in [16]. We introduce the twistor equation in six dimensions as a sufficient condition for the existence of integrable isotropic 3planes. We close with the relation to the existence of Killing spinors and applications in six-dimensional spaces to a family of examples presented in the Kerr-Schild form.

\section{Six-dimensional Newman-Penrose formalism}

We begin by reviewing some aspects of four-dimensional spinors. There the spinor formalism takes full advantage from the fact that the Lorentz group $S O(3,1)$ is isomorphic to $S U(2) \times S U(2)$. Reality conditions relate both factors, so the decomposition of a vector into $S U$ (2) pieces is done via the van der Waerden symbols to a matricial object $A_{\alpha \dot{\alpha}}$. The 
norm of the vector is written as the determinant of the matrix. When it is zero, it means that two rows are linearly dependent, so that

$A^{\alpha \dot{\alpha}}=\kappa^{\alpha} \bar{\kappa}^{\dot{\alpha}}$.

the objects $\kappa^{\alpha}$ and $\bar{\kappa}^{\dot{\alpha}}$ transform as spinors under Lorentz transformations, and they parametrize the null vectors. $\kappa$ and $\bar{\kappa}$ will be the complex conjugate of each other if we want the null vector to be real. The correspondence between spinors and null vectors is up to a multiplicative phase, the "flag" of the spinor, which in itself can be used to encode helicity information, a fact which was very useful for the computation of solutions of arbitrary spin massless fields and their scattering amplitudes.

One can go ahead and substitute the tetrad formalism with spinor language in general relativity. Let $e_{0}, e_{1}, e_{2}, e_{3}$ be a normalized tetrad. The null vectors

$k=\left(e_{0}+e_{1}\right) / \sqrt{2}, \quad l=\left(e_{0}-e_{1}\right) / \sqrt{2}$,

$m=\left(e_{2}+i e_{3}\right) / \sqrt{2}, \quad \bar{m}=\left(e_{2}-i e_{3}\right) / \sqrt{2}$

can be decomposed with two (complex) spinors: $\iota^{\alpha}$ and $o^{\alpha}$ and their complex conjugate:

$k^{\alpha \dot{\alpha}}=l^{\alpha} \bar{l}^{\dot{\alpha}}, \quad l^{\alpha \dot{\alpha}}=o^{\alpha} \bar{o}^{\dot{\alpha}}, \quad m=l^{\alpha} \bar{o}^{\dot{\alpha}}, \quad \bar{m}=o^{\alpha} \bar{l}^{\dot{\alpha}}$.

Note that $k^{a}$ and $l^{a}$ are real whereas $m^{a}$ is the complex conjugate of $\bar{m}^{a}$.

In six dimensions the decomposition of vectors is similar [17]. The symmetry group is $S O(6)$, which we will see as a complex group $S L(4, \mathbb{C})$. One real form of it is the group of isometries of Euclidean space, $S O(6)$, the compact form of $S L(4, \mathbb{C})$ and is isomorphic locally to $S U(4) .{ }^{1}$ The latter has a natural four-dimensional fundamental 4 representation, which can be seen as the spinorial representation of $S O(6)$. Paralleling the four-dimensional case, a six-dimensional vectorial representation can be constructed from the tensor product of the spinorial representation:

$4 \times 4=6+10$

and then a vector can be written as an antisymmetric tensor of spinor indices:

$V^{A B}=\Sigma_{a}^{A B} V^{a}$

where $\Sigma_{a}^{A B}=-\Sigma_{a}^{B A}$ are analogs of the van der Waerden symbols in six dimensions, essentially the positive chirality projection of the Dirac matrices. The inner product of vectors is performed using the completely antisymmetric symbol $\epsilon_{A B C D}$ :

$V^{a} V_{a} \equiv \epsilon_{A B C D} V^{A B} V^{C D}$.

${ }^{1}$ Globally $S U(4)$ a double cover of $S O(6)$.
Now, let us consider a spinorial basis $\kappa_{i}^{A}$, normalized so that $\epsilon_{A B C D} \kappa_{1}^{A} \kappa_{2}^{B} \kappa_{3}^{C} \kappa_{4}^{D}=1$. A generic vector can be expanded in a basis of simple bispinors $\kappa_{i}^{[A} \kappa_{j}^{B]}$, but, because the $\epsilon_{A B C D}$ is completely antisymmetric, the only chance that this vector will not be null is that the expansion involves all of the $\kappa_{i}$. One can then find, for a given null vector $V^{[A B]}$, a spinor basis $\left(\kappa_{i}\right)^{A}, i=1,2,3,4$ such that $V^{[A B]}$ can be decomposed in a bispinor basis involving only three of the $\left(\kappa_{i}\right)^{A}$ :

$$
\begin{aligned}
V^{A B} & =\alpha_{1} \kappa_{1} \wedge \kappa_{2}+\alpha_{2} \kappa_{1} \wedge \kappa_{3}+\alpha_{3} \kappa_{2} \wedge \kappa_{3} \\
& =\left(\kappa_{1}+\frac{\alpha_{3}}{\alpha_{2}} \kappa_{2}\right) \wedge\left(\alpha_{1} \kappa_{2}+\alpha_{2} \kappa_{3}\right)=\chi^{\left[A_{\xi} B\right]},
\end{aligned}
$$

assuming $\alpha_{2} \neq 0$. Then, parallelizing the result in four dimensions, any null vector can be represented as the outer product of spinors, with the converse obviously true, given by (2.6).

While a vector is associated with the exterior product of two spinors, a single spinor $\kappa^{A}$ is associated with an isotropic 3-plane. This 3-plane is generated by the null vectors

$V_{i}^{A B}=\kappa^{[A} \kappa_{i}^{B]}$,

for some basis $\kappa_{i}^{A}$. It is clear that there are three nonzero vectors $V_{i}^{A B}$ and that they generate an isotropic space, since any linear combination of the $V_{i}^{a}$ is a null vector.

A tangent space connection $\nabla_{a}$ can be introduced, and from the zero torsion condition and the Leibniz rule it follows that the following operator is linear on the spinorial fields:

$\left(\nabla_{a} \nabla_{b}-\nabla_{b} \nabla_{a}\right) \kappa^{E}=\mathcal{R}_{a b F}{ }^{E} \kappa^{F}$,

where $a$ and $b$ are coordinate (vector) indices. Since it is antisymmetric under interchange between $a \equiv A E$ and $b \equiv$ $F G$, one can construct from it the spinorial tensor

$R_{A B}{ }^{C D}=\epsilon^{E F G C} \mathcal{R}_{A E F G B}{ }^{D}$,

which is the spinor analog of the Riemann tensor. As in four dimensions, one can decompose it into isometry invariant pieces:

$R_{A B}^{C D}=\Lambda\left(\delta_{A}^{C} \delta_{B}^{D}-4 \delta_{B}^{C} \delta_{A}^{D}\right)+\Phi_{A B}^{C D}+\Psi_{A B}^{C D}$

with $\Lambda$ related to the scalar curvature, $\Phi_{A B}{ }^{C D}=\Phi_{[A B]}{ }^{[C D]}$ with $\Phi_{A C}{ }^{A D}=0$ being the spinor analog of the traceless part of the Ricci tensor, and $\Psi_{A B} C D=\Psi_{(A B)}{ }^{(C D)}$ with $\Psi_{A C} A D=0$ being the spinor analog of the Weyl tensor. The odd form of the first term guarantees that $R_{A B}{ }^{A D}=0$, while $R_{A B}{ }^{C A}=-15 \Lambda \delta_{B}^{C}$. In terms of $S U$ (4) irreducible representations, the decomposition is $\mathbf{1}+\mathbf{2 0}+\mathbf{8 4}$, adding up to 105 independent components of the Riemann tensor in six dimensions. 


\section{The Kerr theorem}

The Kerr theorem gives an implicit solution to all analytic, shear-free, geodetic null congruences in flat space. The problem is strongly tied with the existence of integrable null submanifolds. The connection between the two seemingly distinct problems is deep, as will be discussed in the subsection on generic dimensions. The original result, in four dimensions, can be written in terms of an implicit, analytic function in projective twistor space, and it actually serves as one geometrical interpretation of a twistor [11]. We will see that most of the geometrical picture translate to six dimensions.

We will follow the discussion in [18], taking a vectorial point of view. This problem has also been considered using spinorial techniques for the general even-dimensional case by [3]. For a more recent consideration see [19]. Let us begin with a flat six-dimensional flat space with Lorentzian signature:

$d s^{2}=d u d v+d z_{1} d \bar{z}_{1}+d z_{2} d \bar{z}_{2}$.

The null 1-forms

$e_{0}=d u+\bar{Y}^{i} d z_{i}+Y^{i} d \bar{z}_{i}-Y_{i} \bar{Y}^{i} d v, \quad e_{i}=d z_{i}-\bar{Y}_{i} d v$

will determine an integrable distribution (submanifold) if their brackets all close, or, in the dual formulation of Frobenius' theorem, if $d e^{i}$ can be written as a linear combination of $e^{i} \wedge e^{j}$ tangent to the submanifold. A somewhat tedious computation yields, for $d e_{0} \wedge e_{0} \wedge e_{1} \wedge e_{2}=0$, the following equations:

$$
\begin{aligned}
& \partial_{v} Y^{1}+Y^{2} \partial_{2} Y^{1}+Y^{1} \partial_{1} Y^{1}-Y^{1} \bar{Y}^{1} \partial_{u} Y^{1}-Y^{1} \bar{Y}^{2} \partial_{u} Y^{2} \\
& \quad+\bar{Y}^{1} \bar{\partial}_{1} Y^{1}+\bar{Y}^{2} \bar{\partial}_{1} Y^{2}=0, \\
& \partial_{v} Y^{2}+Y^{2} \partial_{2} Y^{2}+Y^{1} \partial_{1} Y^{2}-Y_{2} \bar{Y}^{1} \partial_{u} Y^{1}-Y^{2} \bar{Y}^{2} \partial_{u} Y^{2} \\
& \quad+\bar{Y}^{1} \bar{\partial}_{2} Y^{1}+\bar{Y}^{2} \bar{\partial}_{2} Y^{2}=0, \\
& \bar{\partial}_{2} Y^{1}-\bar{\partial}_{1} Y^{2}+Y^{1} \partial_{u} Y^{2}-Y^{2} \partial_{u} Y^{1}=0, \\
& Y^{2}\left(\partial_{v} Y^{1}+\bar{Y}^{2} \bar{\partial}_{1} Y^{2}-Y^{1} \partial_{2} Y^{2}+Y^{2} \partial_{2} Y^{1}+\bar{Y}^{1} \bar{\partial}_{1} Y^{1}\right) \\
& \quad-Y^{1}\left(\partial_{v} Y^{2}+\bar{Y}^{1} \bar{\partial}_{2} Y^{1}+Y^{1} \partial_{1} Y^{2}-Y^{2} \partial_{1} Y^{1}+\bar{Y}^{2} \bar{\partial}_{2} Y^{2}\right)=0,
\end{aligned}
$$

and the equations following from the requisition that $d e_{1} \wedge$ $e_{0} \wedge e_{1} \wedge e_{2}$ and $d e_{2} \wedge e_{0} \wedge e_{1} \wedge e_{2}$ vanish:

$$
\begin{gathered}
\bar{\partial}_{1} Y^{i}-Y^{1} \partial_{u} Y^{i}=0, \quad \bar{\partial}_{2} Y^{i}-Y^{2} \partial_{u} Y^{i}=0, \\
Y^{2} \bar{\partial}_{1} Y^{i}-Y^{1} \bar{\partial}_{2} Y^{i}=0,
\end{gathered}
$$

with $i=1,2$. Note that the first two equations imply the third one.

Despite the coupling, the equations are simple to solve. After some manipulations, they result in

$$
\left(\bar{\partial}_{i}-Y^{i} \partial_{u}\right) Y^{j}=0, \quad\left(\partial_{v}+\sum_{i} Y^{i} \partial_{i}\right) Y^{j}=0, \quad i=1,2,
$$

and their complex conjugate. These equations can be solved by the method of characteristics [20]. The solution is given implicitly in terms of a general function $F$ of five complex arguments:

$F\left(Y^{1}, Y^{2} ; v Y^{1}-z_{1}, v Y^{2}-z_{2} ; u+\bar{z}_{1} Y^{1}+\bar{z}_{2} Y^{2}\right)=0$.

This version of the solution of integrable distributions is more economical than the general expression given in [3], but the spinorial interpretation is less clear. In order to recover it, let us introduce the spinors

$\kappa^{A}=\left[\begin{array}{c}Y^{1} \\ Y^{2} \\ 0 \\ 1\end{array}\right], \quad \bar{\kappa}^{A}=\left[\begin{array}{c}\bar{Y}^{2} \\ -\bar{Y}^{1} \\ 1 \\ 0\end{array}\right]$,

which generate the null vector $k^{a} \equiv k^{[A B]}$ by the exterior product $k^{A B}=\kappa^{[A} \bar{\kappa}^{B]}$. Note that $\bar{\kappa}^{A}$ is related to the complex conjugate of $\kappa^{A}$ via a conjugation operator:

$\bar{\kappa}^{A}=B^{A B} \kappa_{B}^{*}, \quad B=\left[\begin{array}{cc}i \sigma^{2} & 0 \\ 0 & i \sigma^{2}\end{array}\right]=1 \otimes i \sigma^{2}$,

so that the vector $k^{a}$ is real.

Lastly, we introduce the "position vector" $x_{A B}$, an antisymmetric matrix in spinor space:

$x_{A B}=\left[\begin{array}{cccc}0 & v & -\bar{z}_{1} & -z_{2} \\ -v & 0 & -\bar{z}_{2} & z_{1} \\ \bar{z}_{1} & \bar{z}_{2} & 0 & u \\ z_{2} & -z_{1} & -u & 0\end{array}\right]$.

The attribution of the positions of the coordinates is of course arbitrary. There is a $S U(2) \times S U(2) \simeq S O(4)$ isometrythe "little group"-in spinor space which keeps the vector invariant. We will discuss its geometrical interpretation. We recast (3.6) in an expression using spinors:

$F\left(\kappa^{A}, x_{C B} \kappa^{B}\right)=0$,

where $F\left(\kappa^{A}, \zeta_{B}\right)$ is a holomorphic function from $\mathbf{4} \times \overline{\mathbf{4}}$ to the complex numbers. The pair $Z^{\mathcal{I}}=\left(\kappa^{A}, \zeta_{B}\right)$ is a sixdimensional twistor. From the construction above, the function $F$ needs only be defined for $\kappa^{A} \zeta_{A}=0$, so one defines in twistor space a natural pairing:

$$
\left\langle Z^{\mathcal{I}}, U^{\mathcal{J}}\right\rangle \equiv\left\langle\left(\lambda^{A}, \chi_{B}\right),\left(\pi^{C}, \zeta_{D}\right)\right\rangle=\lambda^{A} \zeta_{A}+\pi^{D} \chi_{B} .
$$

With the choice of conjugation $\bar{Z}^{\mathcal{I}}=\left(\bar{\kappa}^{A}, \bar{\zeta}_{B}\right), \bar{\zeta}_{B}=$ $\left(B^{-1}\right)_{B C}\left(\zeta^{*}\right)^{C}$, this form has signature $(6,2)$. 
The crucial fact in six dimensions is that now $F$ has the projective property:

$F\left(\alpha \kappa^{A}+\beta \bar{\zeta}^{A}, \alpha \zeta_{B}+\beta \bar{\kappa}_{B}\right)=F\left(\kappa^{A}, \zeta_{B}\right)$.

These transformations keep the vector $k^{A B}=\kappa^{[A} \bar{\kappa}^{B]}$ invariant. This property shows that the relation between twistors and geodesic, shear-free null vector fields is not bijective, as in [3]. There are many isotropic 3-planes which give rise to the same integrable vector field. This contrasts to the situation in $3+1$ dimensions, where, given a real null vector field, all isotropic planes containing it are related by a phase. It should also be pointed out that without the reality condition enforced by the operator $B$, the solution $F$ has to be thought of as a projective section on the Grassmanian space $\operatorname{Gr}(2,4)$, which, unlike its four-dimensional sibling, is not a projective space.

Incidentally, in the six-dimensional case the antisymmetric operator $x_{A B}$ is not determined uniquely by $\kappa^{A}$ and $\zeta_{A}$, since the spinor space has more than two dimensions. The notion that the spacetime coordinates arise from the "more fundamental" objects, the twistors, cannot work as in four dimensions.

\subsection{Six-dimensional helicity spinors}

The results above encode an underlying algebraic structure. The six-dimensional Lorentzian space considered also shows up in the construction of instantons for four-dimensional Yang-Mills theories, where the space of solutions can be seen as the twistor space for Euclidean four-dimensional spacetime [21]. The construction is relevant for our case. Let us recall that the twistor space in four dimension is defined, e.g. in [11], as the pair of 2-spinors

$Z^{a}=\left(\omega^{\alpha}, \pi_{\dot{\beta}}\right)$ with a natural linear symmetric inner product of signature $(2,2)$ :

$\left\langle Z_{1}, Z_{2}\right\rangle=\left(\omega_{2}\right)^{\alpha}\left(\bar{\pi}_{1}\right)_{\alpha}+\left(\bar{\omega}_{1}\right)^{\dot{\alpha}}\left(\pi_{2}\right)_{\dot{\alpha}}$.

The group that keeps this structure invariant is another real form of $S L(4, \mathbb{C}), S U(2,2)$, which is isomorphic to $S O(4,2)$. For $S O(5,1)$, the accidental isomorphism is with $S L(2, \mathbb{H})$, or $2 \times 2$, unit-determinant matrices over the quaternion field $\mathbb{H}[21,22]$. Thus, spinor space in six dimensions is essentially another real form of twistor space in four dimensions. Given four complex numbers $\left\{z_{1}, z_{2}, z_{3}, z_{4}\right\}$, one can embed them into a pair of quaternions as follows:

$\left(z_{1}, z_{2}, z_{3}, z_{4}\right) \rightarrow\left(z_{1}+j z_{2}, z_{3}+j z_{4}\right)$,

with $j \neq i$ a basis element of the quaternions.

In the quaternion language, the symmetry

$$
\left(\begin{array}{c}
\kappa^{A} \\
\zeta_{B}
\end{array}\right) \equiv\left(\begin{array}{c}
\alpha \kappa^{A}+\beta \bar{\zeta}^{A} \\
\alpha \zeta_{B}+\beta \bar{\kappa}_{B}
\end{array}\right)
$$

corresponds to right multiplication by a quaternion $q=$ $\alpha+\beta j$ [22]. Left and right multiplication from unit-norm quaternions make for a $\mathrm{SU}(2) \times \mathrm{SU}(2) \sim \mathrm{SO}(4)$ symmetry that acts on the spinor $\kappa^{A}$ but that leaves the null vector $\kappa^{[A} \bar{\kappa}^{B]}$ invariant. In terms of the particular spinor representation given in (3.8), the right action is of the form $g \otimes i \sigma^{2}$, $g \in \mathrm{SU}(2)$, the generic operator which commutes with $B$. This symmetry is the "little group" of $S O(5,1)$, and, among the multiple spinors which represent the same null vector $k^{A B}$, one line is chosen by representing the helicity as a spacelike vector orthogonal to $k^{[A B]}$. The function representing the solution of the Kerr theorem is actually a function on $\mathbb{H P}^{1}$, the projective quaternionic line [21].

To each isotropic and simple 3-plane one associates a spinor: given a null basis $\left\{e^{1}, e^{2}, e^{3}\right\}$ of the 3-plane, one constructs a basis of spinors $\left(\kappa_{i}\right)^{A}$ such that $e^{i}=\left(\kappa_{0}\right)^{[A}\left(\kappa_{i}\right)^{B]}$ for each $i=1,2,3$. Following the treatment in [17], given a spinor $\kappa^{A}$, there is only one isotropic 3-plane associated to it: the 3-form $T_{a b c} \equiv T_{[A B][C D][E F]}$ obtained by inverting the relation

$T_{A B C D E F} \epsilon^{D E F G}=\bar{T}_{A[B} \delta_{C]}^{G}+\tilde{T}^{F G_{\epsilon}} \epsilon_{A B C F}$

defines a generic 3 -plane for symmetric $\bar{T}_{A B}$ and $\tilde{T}^{A B}$. If one has either of them simple, and the other zero, like $\bar{T}_{A B}=0$ and $\tilde{T}^{A B}=\kappa^{A} \kappa^{B}$, the 3-plane is isotropic (and self-dual). This 3 -plane contains the null vector $k^{a}=\kappa^{[A} \bar{\kappa}^{B]}$. The vector is actually in the intersection of the 3-planes associated with $\kappa^{A}$ and $\bar{\kappa}^{A}$.

In order to define a helicity of a twistor, take the pair $\left(\kappa^{A}, \zeta_{B}\right)$ and construct from it the 2-plane:

$S^{[A B][C D]}=\kappa^{[A} \epsilon^{B] C D E} \zeta_{E}-\kappa^{[C} \epsilon^{D] E B C} \zeta_{E}$.

Because of the null condition $\kappa^{A} \zeta_{A}=0$, this plane is simple and contains the vector $k^{a}=\kappa^{[A} \bar{\kappa}^{B]}$. The other vector can be extracted with the following procedure. Define the rest of the spinor basis $\pi$ and $\bar{\pi}$ such that

$\kappa^{[A} \bar{\kappa}^{B} \pi^{C} \bar{\pi}^{D]}=\epsilon^{A B C D}$.

This essentially means that $\pi^{A}$ is dual to $\zeta_{A}$. We have the helicity in the form of the vector

$m^{a}=\kappa^{[A} \pi^{B]}+\bar{\kappa}^{[A} \bar{\pi}^{B]}$.

Likewise, one can associate with the coordinates of $\mathbb{H} \mathbb{P}^{1}$ the null vectors and all helicity vectors associated with the little group.

Given this, one can reinterpret formulas given in [7] as holomorphic projective sections in twistor space, or $\mathbb{H P}^{1}$. It would be interesting if one could use analytic properties to define n-point functions of six-dimensional Lorentz invariant massless field theories, as in [23-25]. The construction seems similar to that of [16], but with the extra geometric interpretation. 


\subsection{A digression in generic (even) dimensions}

In a flat, even-dimensional Lorentzian manifold of null coordinates $\left\{u, v, z^{i}, \bar{z}^{i}\right\}$, the analog of (3.5) is

$$
\begin{aligned}
& \left(\bar{\partial}_{i}-Y^{i} \partial_{u}\right) Y^{j}=0, \quad\left(\partial_{v}+\sum_{i} Y^{i} \partial_{i}\right) Y^{j}=0, \\
& \quad i, j=1, \ldots,(D-2) / 2
\end{aligned}
$$

and their complex conjugate. Given the vector fields

$$
\begin{aligned}
& k^{a}=\frac{\partial}{\partial v}+Y^{i} \frac{\partial}{\partial z^{i}}+\sum_{i} \bar{Y}^{i}\left(m^{i}\right)^{a}, \\
& \left(m^{i}\right)^{a}=\frac{\partial}{\partial \bar{z}^{i}}-Y^{i} \frac{\partial}{\partial u} .
\end{aligned}
$$

We can see that (3.20) are equivalent to the vanishing of the "anti-holomorphic" part of the (flat space covariant) derivative of $k^{a}$ :

$\left(m^{i}\right)^{b}\left(m^{j}\right)^{a} \partial_{a} k_{b}=0, \quad i, j=1, \ldots,(D-2) / 2$,

which in turn means that the attribution of "holomorphic" and "anti-holomorphic" parts for the space generated by the $m^{i}$ and $\bar{m}^{i}$ is maintained under parallel transport by $k^{a}$.

The solution of (3.20) can also be obtained from the method of characteristics. The result is an implicit function of $D-1$ complex arguments:

$F\left(\left\{Y^{i}\right\} ;\left\{v Y^{i}-z_{i}\right\} ; u+\sum_{i} \bar{z}_{i} Y^{i}\right)=0 ;$

one sees that the number of arguments is much smaller than the number of components of pure spinors in $D$ dimensions [3]. This should be seen as a function of the Grassmann plane $\operatorname{Gr}(2, D-2)$ in spinorial space, so the generic function of the pure spinor has to be modded out by the subset of the isometries of $S O(D-1,1)$ which leave $\operatorname{Gr}(2, D-2)$ invariant. These spaces are not projective, so the twistor analysis has to be carried over using other methods. This shows that real space applications of twistor methods in higher dimensions are not trivial, and the generic analysis based on (complex) pure spinors is incomplete.

\section{Six-dimensional twistors}

As seen above, an isotropic 3-plane is represented by a simple 3-form $T_{a b c}=T_{[a b c]}=e^{1} \wedge e^{2} \wedge e^{3}$, whose translation to the spinor language is the product $\kappa^{A} \kappa^{B}$, with $e^{i} \equiv \kappa^{[A}\left(\kappa_{i}\right)^{B]}$. Frobenius' theorem states that this plane will be integrable if the 3-form $T_{a b c}$ is, up to scale, a harmonic (closed and co-closed). ${ }^{2}$ Since an isotropic 3-plane is necessarily selfdual, then one needs only to check for closedness. In terms

\footnotetext{
${ }^{2}$ In this guise the theorem is sometimes called Mariot-Robinson's.
}

of spinors, this condition is

$\nabla_{A B}\left(\kappa^{A} \kappa^{C}\right)=0$,

or

$\kappa^{A} \nabla_{A B} \kappa^{C}=-\left(\nabla_{A B} \kappa^{A}\right) \kappa^{C}$.

The six-dimensional twistor equation

$\nabla_{A B} \kappa^{C}=\pi_{[A} \delta_{B]}^{C}$

solves the constraint if $\pi_{A} \kappa^{A}=0$. The twistor equation is the only plausible simplification for the derivative of a spinor. In general, the derivative of $\kappa^{A}$ will have the following expansion:

$\nabla_{A B} \kappa^{C}=\pi_{[A} \delta_{B]}^{C}+\Theta_{A B}^{C}$,

with $\Theta_{A B}^{C}$ in the 20 of $S U$ (4) being antisymmetric in the lower indices and traceless. We impose the condition that $\Theta_{A B}^{C}=0$ as the only Lorentz-invariant condition that leaves the right number of degrees of freedom involved in the Kerr theorem.

The six-dimensional twistor (4.3) has the usual integrability condition stemming from the Ricci identity:

$\left(\nabla_{A B} \nabla_{C D}-\nabla_{C D} \nabla_{A B}\right) \kappa^{E}=\mathcal{R}_{A B C D F}{ }^{E} \kappa^{F}$.

Contracting with the antisymmetric tensor, one obtains

$\epsilon^{B C D G}\left(\nabla_{A B} \nabla_{C D}-\nabla_{C D} \nabla_{A B}\right) \kappa^{E}=R_{A F}^{G E} \kappa^{F}$,

which, using (4.3) and rearranging the indices, can be written as

$$
\begin{aligned}
& 3 \epsilon^{C B E G} \nabla_{\left[A B \pi_{C]}\right.}+2 \epsilon^{B C E G} \nabla_{B C} \pi_{A}-\epsilon^{C D B G} \delta_{A}^{E} \nabla_{C D} \pi_{B} \\
& =2 R_{A F}{ }^{G E} \kappa^{F} .
\end{aligned}
$$

Contracting $A$ and $E$, and using $R_{A F}{ }^{G A}=-15 \Lambda \delta_{F}^{G}$ (2.11), we find that

$\epsilon^{A B C D} \nabla_{A B} \pi_{C}=6 \Lambda \kappa^{D}$.

with $\Lambda$ related to the scalar curvature. For Einstein manifolds $\Phi_{A F}{ }^{E F}=0, \Lambda$ is a constant and the antisymmetric part of the left hand side imposes a dual twistor equation for $\pi_{A}$ :

$\nabla^{A B} \pi_{C}=2 \Lambda \delta_{C}^{[A} \kappa^{B]}$.

The twistor pair $\left(\kappa^{A}, \pi_{B}\right)$ then solves the zero-mass half-spin (Weyl) equations. The symmetric part of $E G$ in (4.7) gives an algebraic condition on the Weyl spinor:

$\Psi_{A F}^{E G_{\kappa} F}=0$.

Because of (4.9), $\pi_{c}$ obeys a similar condition: $\Psi_{A F} E G_{\pi_{G}}=$ 0 . In quaternionic language, the twistor $\left(\kappa^{A}, \pi_{C}\right)$ is an eigenvector of the (symmetric) Weyl operator with zero eigenvalue. 
If the manifold has a vanishing Riemann tensor, then $\pi_{C}$ is covariantly constant and therefore the twistor Eq. (4.3) can be solved as

$\kappa^{A}=\psi^{A}+x^{A B} \lambda_{B}$

with $\psi^{A}$ and $\lambda_{B}$ constant spinors. This is an exact analog of the four-dimensional case. Like its four-dimensional sibling, it can be defined as the null subspace of the twistor space, where the metric is the usual pairing:

$\left\langle\left(\pi^{A}, \lambda_{B}\right),\left(\chi^{C}, \zeta_{D}\right)\right\rangle=\pi^{A} \zeta_{A}+\lambda_{B} \chi^{B}$.

With the choice of charge conjugation operator defining a real form in the eight-dimensional complex space spanned by the twistors.

For generic Einstein manifolds, the pair $Z^{\mathcal{I}}=\left(\psi^{A}, \chi_{B}\right)$ has the interpretation of a Killing spinor [15]. The twistor equation and the corresponding dual (4.9) are written with the help of gamma matrices as

$\nabla \eta=c R \eta$.

This holds for some constant $c$, and $R$ being the scalar curvature. One then sees that, as in four dimensions, the algebraic property that $\kappa^{A}$ is annihilated by the Weyl spinor-a principal spinor-is imperative for the existence of solutions to (4.13).

Given that one can associate spinors to integrable harmonic 3-planes, the number of solutions to the twistor equation is a topological invariant, the intersection number (Betti number for half-dimensional submanifolds). Being harmonic, the forms can be seen to saturate the Green inequality. This property can be generalized to "impure spinors", which are related to lower-dimensional isotropic manifolds, and they can be used to define "calibrations" [26] and are at the heart of the saturation of inequalities that allow for computations in the so-called "attractor mechanism" [27].

\subsection{A family of examples}

There are a family of spaces allowing for a principal spinor. These are the Kerr-Schild spaces, whose metric is given by

$g_{a b}=\bar{g}_{a b}-2 S k_{a} k_{b}$

where $\bar{g}_{a b}$ is a maximally symmetric metric (for $d S_{6}, A d S_{6}$ or $\left.\mathbb{R}^{(5,1)}\right), k_{a}$ is a geodesic, shear-free null vector field, with respect to $\bar{g}_{a b}$, and $S$ is a function. These metrics have been studied extensively in four dimensions [18,28,29]. The treatment below will follow [17] closely. Solutions of Einstein's equations of this form in four dimensions include all pp-wave backgrounds, as well as the Kerr-Newman family of black holes. The properties that $k^{a}$ is geodesic and shear-free in the metric $\bar{g}_{a b}$ translate to the metric $g_{a b}$. We will assume that $D S=k^{c} \nabla_{c} S=0$. If $C_{a b}^{c}$ is the relative connection between the derivatives $\nabla_{a}$ and $\bar{\nabla}_{a}$, associated, respectively, to the metrics $g_{a b}$ and $\bar{g}_{a b}$, we have

$$
\begin{aligned}
C_{a b}^{c}= & \left(\bar{\nabla}_{a} S\right) k^{d} k_{b}+\left(\bar{\nabla}_{b} S\right) k^{d} k_{a} \\
& -\left(\bar{\nabla}^{d} S\right) k_{a} k_{b}+2 S(D S) k^{c} k_{a} k_{b} .
\end{aligned}
$$

Indices are raised with the maximally symmetric metric $\bar{g}_{a b}$. These satisfy $C_{a b}^{c} k^{b}=0$, so if $k^{a}$ is geodesic with respect to $\nabla_{a}$, it will also be so with respect to $\bar{\nabla}_{a}$. Moreover, since $\bar{g}_{a b}$ is maximally symmetric, it is conformally flat and the integrability properties of $k^{a}$ which make for the hypothesis of the Kerr theorem remain valid, at least locally. The $k^{a}$ can be thought of as the real part of an integrable 3-plane, as in Sect. 3 .

With a little effort, one can compute the Riemann tensor associated with $g_{a b}$ :

$R_{a b c}{ }^{d}=\bar{R}_{a b c}{ }^{d}-\bar{\nabla}_{a} C_{b c}^{d}+\bar{\nabla}_{b} C_{a c}^{d}+C_{a c}^{e} C_{e b}^{d}-C_{b c}^{e} C_{e a}^{d}$,

with $\bar{R}_{a b c}^{d}$ maximally symmetric. The result is

$$
\begin{aligned}
R_{a b c d}= & \bar{R}_{a b c d}-\frac{R}{15} S\left(k_{[a} \bar{g}_{b] c} k_{d}-k_{[a} \bar{g}_{b] d} k_{c}\right) \\
& -2 k_{[a}\left(\bar{\nabla}_{b]} \nabla_{c} S\right) k_{d}-2 k_{[a}\left(\bar{\nabla}_{b]} \nabla_{d} S\right) k_{c} .
\end{aligned}
$$

The Ricci tensor is

$R_{a b}=\bar{R}_{a b}-\Delta S k_{a} k_{b}$

where $\Delta$ is the Laplacian in the transverse space, related to $\bar{g}_{a b}$. We will assume that the space is Einsteinian. Given the form of the Riemann tensor, one can show that the Weyl tensor has the property

$C_{a b c d} k^{b} k^{d}=0$,

which can be translated to the spinorial language:

$\Psi_{A E}^{I J}=C_{[A B][C D][E F][G H]} \epsilon^{B C D I} \epsilon^{F G H J}$.

Now, we write $k^{a}=\kappa^{[A} \bar{\kappa}^{B]}$, for an integrable $\kappa^{A}$ satisfying (4.3). We choose a spinorial basis such that $\epsilon^{A B C D}=$ $\kappa^{[A} \bar{\kappa}^{B}\left(\pi^{*}\right)^{C}\left(\bar{\pi}^{*}\right)^{D]}$ as in Sect. 3.1. Here the asterisk denotes dual. Given that the dual spinor $\pi_{B}$ satisfies a similar Eq. (4.9), we will also assume that it is integrable. We introduce the dual basis $\left\{\left(\kappa^{*}\right)_{A},\left(\bar{\kappa}^{*}\right)_{A}, \pi_{A}, \bar{\pi}_{A}\right\}$, and we note that $k_{a}=\pi_{[A} \bar{\pi}_{B]}$. We find that (4.19) means

$\Psi_{A E}^{I J} \pi_{[I} \bar{\pi}_{B]} \pi_{[J} \bar{\pi}_{F]}=0$.

A similar calculation with the $\pi_{E}$ results in

$\Psi_{A E}^{I J} \kappa^{[A} \bar{\kappa}^{H]} \kappa^{[E} \bar{\kappa}^{K]}=0$.

These identities constrain the form of the Weyl spinor so that $\Psi_{A E}{ }^{I J} \kappa^{E}=0$. The differential equation for $S$ can be solved by usual methods, and the $\kappa^{A}$ are taken from the general solution in flat space (3.6). 
As in four dimensions, this family of spacetimes can cover all known families of reduced holonomy manifolds. Some of those, like the cone of Einstein-Sasaki's [30], can be obtained from a Wick rotation of Lorentzian manifolds. However, there seem to be more parameters encoded in the $\kappa^{A}$ as in (3.10), and the constants fixing $S$, than the usual $p, q, r$ involved in the $L^{(p, q, r)}$ and the Reeb vector. Although it should be remembered that in the Euclidean case one would like to impose the condition that the orbits of the vector $k^{a}$ are closed. We hope to address this issue in the future.

\section{Conclusions}

In this letter we addressed the relationship between Kerr theorem and pure spinors in six dimensions. We showed that, while the spinorial language is natural to talk about solutions to geodesic, shear-free, null vector fields, the correspondence is not one-to-one, which is reminiscent of the quaternionic structure of supersymmetry in six dimensions [22]. We then turned to the problem of the twistor equation in six dimensions, and we showed that, just like the four-dimensional case [15], the Killing spinor equation can quite naturally be cast in the Newman-Penrose language. Finally, we presented a generalization of Kerr-Schild metrics which display the algebraic property allowing for solutions of the twistor equation.

A great deal of applications of pure spinors have been put forward in the last ten years, mainly to the covariant superstring [31]. The effort has been basically centered on algebraic aspects of pure spinors. However, most aspects of such applications have had their birth in geometrical aspects of four-dimensional general relativity. This work is the result of some effort spent in trying to understand pure spinors from a geometrical point of view in six dimensions. In eight or more dimensions, the geometrical point of view becomes more natural, as the algebraic one becomes more mysterious: for instance, the pure condition becomes a quadratic constraint on the spinors, while the relationship to maximal-dimension isotropic planes continues to hold. In six dimensions, as we can see here, many of the features translate quite naturally to the new setting. We hope that the fresh point of view will be useful to other people.

Acknowledgments The author is indebted to Carlos Batista and Amílcar de Queiroz for a critical and thorough reading of the manuscript, and he would also like to thank A. P. Balachandran and Fábio Novaes for comments and suggestions.

Open Access This article is distributed under the terms of the Creative Commons Attribution License which permits any use, distribution, and reproduction in any medium, provided the original author(s) and the source are credited.

Funded by $\mathrm{SCOAP}^{3}$ / License Version CC BY 4.0.

\section{References}

1. R. Penrose, W. Rindler, Spinors and Space-time: Two Spinor Calculus and Relativistic Fields, vol. 1. (Cambridge University Press, Cambridge, 1987)

2. R. Penrose, W. Rindler, Spinors and Space-time, Spinor and Twistor Methods in Space-Time Geometry, vol. 2. (Cambridge University Press, Cambridge, 1988)

3. L. Hughston, L. Mason, A generalized Kerr-Robinson theorem. Class. Quant. Grav. 5, 275-285 (1988)

4. L. Hughston, in The Wave Equation in Even Dimensions. Further Advances in Twistor Theory, Research Notes in Mathematics 231, vol. 1 (1990), p. 26

5. L. Hughston, in Further Advances in Twistor Theory. A Remarkable Connection Between The Wave Equation and Pure Spinors in Higher Dimensions. Research Notes in Mathematics 231, vol. 1 (1990), p. 37

6. A. Kurusa, A Characterization of the Radon transform's range by a system of PDEs. J. Math. Anal. Appl. 161, 218-226 (1991)

7. N. Berkovits, S.A. Cherkis, Higher-dimensional twistor transforms using pure spinors. JHEP 0412, 049 (2004). [hep-th/0409243]

8. A.H. Chamseddine, S. Ferrara, G.W. Gibbons, R. Kallosh, Enhancement of supersymmetry near 5-d black hole horizon. Phys. Rev. D 55, 3647-3653 (1997). [hep-th/9610155]

9. B. Carneiro da Cunha, A. de Queiroz, Killing Horizons and Spinors. arXiv: 1312.4911

10. R.M. Wald, General Relativity (The University of Chicago Press, Chicago, 1984)

11. S. A. Huggett, K.P. Tod, An Introduction to Twistor Theory (Cambridge, Cambridge University Press, 1994)

12. R.S. Ward, R.O. Wells, Twistor Geometry and Field Theory. (Cambridge, Cambridge University Press, 1990)

13. E. Cartan, The Theory of Spinors (Dover, New York, 1981)

14. C. Chevalley, The Algebraic Theory of Spinors (Columbia University Press, New York, 1954)

15. P. van Nieuwenhuizen, N. Warner, Integrability conditions for Killing spinors. Commun. Math. Phys. 93, 277 (1984)

16. C. Cheung, D. O'Connell, Amplitudes and spinor-helicity in six dimensions. JHEP 0907, 075 (2009). [arXiv:0902.0981]

17. C. Batista, B. Carneiro da Cunha, Spinors and the Weyl Tensor Classification in Six Dimensions, arXiv:1212.2689

18. H. Stephani, D. Kramer, M.A. MacCallum, C. Hoenselaers, E. Herlt, Exact solutions of Einstein's field equations (Cambridge, Cambridge University Press, 2003)

19. A. Taghavi-Chabert, Pure Spinors, Intrinsic Torsion and Curvature in Even Dimensions, arXiv:1212.3595

20. F. John, in Partial Differential Equations. Applied Mathematical Sciences, vol. 1, 3rd edn. (Springer, Berlin, 1991)

21. M. Atiyah, Geometry of Yang-Mills Fields, Pisa, Italy. Sc Norm. Sup (1979)

22. T. Kugo, P.K. Townsend, Supersymmetry and the division algebras. Nucl. Phys. B 221, 357 (1983)

23. S. Weinberg, Six-dimensional methods for four-dimensional conformal field theories. Phys. Rev. D 82, 045031 (2010). [arXiv:1006.3480]

24. S. Weinberg, Six-dimensional methods for four-dimensional conformal field theories II: irreducible fields. Phys. Rev. D 86, 085013 (2012). [arXiv:1209.4659]

25. C. Saemann, M. Wolf, On twistors and conformal field theories from six dimensions. J. Math. Phys. 54, 013507 (2013). [arXiv:1111.2539]

26. F.R. Harvey, Spinors and Calibrations, Perspectives in Mathematics, vol. 9 (Academic Press, New York, 1990) 
27. A. Sen, Black hole entropy function, attractors and precision counting of microstates. Gen. Rel. Grav. 40, 2249-2431 (2008) [arXiv:0708.1270]

28. M. Gurses, F. Gursey, Lorentz covariant treatment of the kerr-schild geometry. J. Math. Phys. 16(12), 2385 (1975)

29. M. Ortaggio, V. Pravda, A. Pravdova, Higher dimensional KerrSchild spacetimes. Class. Quant. Grav. 26, 025008 (2009), [arXiv:0808.2165]
30. D. Martelli, J. Sparks, S.-T. Yau, Sasaki-Einstein manifolds and volume minimisation. Commun. Math. Phys. 280, 611-673 (2008). [hep-th/0603021]

31. N. Berkovits, Super Poincare covariant quantization of the superstring. JHEP 0004, 018 (2000). [hep-th/0001035] 